\title{
The NEMO 3 Experiment Results
}

\author{
Ladislav Vála*† \\ IEAP, Czech Technical University in Prague, Czech Republic \\ E-mail: ladislav.vala@utef.cvut.cz
}

The NEMO 3 detector, which is devoted to searching for neutrinoless double beta decay (0v $\beta \beta)$, has been operating in the Fréjus Underground Laboratory in France since February 2003. Halflives of the two-neutrino double beta decays $(2 v \beta \beta)$ have been measured for ${ }^{100} \mathrm{Mo}$ and ${ }^{82} \mathrm{Se}$. After 389 days of data collection from February 2003 until September 2004, no evidence for neutrinoless double beta decay was found from $7 \mathrm{~kg}$ of ${ }^{100} \mathrm{Mo}$ and $1 \mathrm{~kg}$ of ${ }^{82} \mathrm{Se}$. The corresponding lower limits for the half-lives are $4.6 \times 10^{23} \mathrm{y}$ at $90 \%$ C.L. for ${ }^{100} \mathrm{Mo}$ and $1.0 \times 10^{23} \mathrm{y}$ at $90 \%$ C.L. for ${ }^{82} \mathrm{Se}$. Limits for the effective Majorana neutrino mass are $\left\langle m_{v}\right\rangle<0.66-2.81 \mathrm{eV}$ for ${ }^{100} \mathrm{Mo}$ and $\left\langle m_{v}\right\rangle<1.75-4.86 \mathrm{eV}$ for ${ }^{82} \mathrm{Se}$. Radon was the dominant background and is now strongly supressed by a radon-trapping facility, which has been in operation since October 2004. The expected sensitivity of the NEMO 3 experiment after five years of data collection will be $T_{1 / 2}^{0 v \beta \beta}>2 \times 10^{24}$ y at $90 \%$ C.L. for ${ }^{100} \mathrm{Mo}$ and $8.3 \times 10^{23}$ y at $90 \%$ C.L. for ${ }^{82} \mathrm{Se}$, corresponding to $\left\langle m_{v}\right\rangle<0.3-1.3 \mathrm{eV}$ and $\left\langle m_{v}\right\rangle<0.6-1.7 \mathrm{eV}$ respectively.

International Europhysics Conference on High Energy Physics

July 21st - 27th 2005

Lisboa, Portugal

\footnotetext{
* Speaker.

† On behalf of the NEMO 3 Collaboration.
} 


\section{Introduction}

Neutrinoless double decay $(0 v \beta \beta)$ is a process which violates lepton number by two units, and is a probe for physics beyond the Standard Model. The observation of this decay would prove that neutrinos are Majorana particles, it would also constrain the mass spectrum and the absolute mass of the neutrinos. The NEMO 3 detector is devoted to the search for $0 v \beta \beta$ decay by means of the direct detection of the two electrons emitted in double beta decay. NEMO 3 combines a tracking detector with a calorimeter for simultaneous particle detection and energy measurements.

\section{The NEMO 3 detector}

The NEMO 3 experiment is located in the Fréjus Underground Laboratory, the $\mathrm{LSM}^{1}$, at the depth of $4800 \mathrm{~m}$ water equivalent. The detector is cylindrical in design, it is divided into twenty equal sectors, and its external dimensions (with shields) are about $6 \mathrm{~m}$ in diameter and $4 \mathrm{~m}$ in height. NEMO 3 combines two detection techniques: particle identification provided by a wire tracking chamber and energy and time measurements of particles with a calorimeter. Thanks to the combination of these techniques the NEMO 3 detector is capable of identifying electrons, positrons, photons, and $\alpha$-particles by unambiguous topological signatures.

The wire chamber is made of 6180 open octagonal drift cells operating in Geiger mode (Geiger cells). A gas mixture of $\sim 95 \%$ helium, $4 \%$ ethyl-alcohol, $1 \%$ argon and $0.15 \%$ water at 10 mbar above atmospheric pressure is used as the filling gas. Each cell provides a three-dimensional measurement of the charged particle tracks by recording the drift time and the two plasma propagation times.

The calorimeter, which surrounds the wire chamber, is composed of 1940 plastic scintillators coupled by light-guides to very low-radioactivity photomultiplier tubes (PMTs) from Hammamatsu. The energy resolution $\sigma_{E} / E$ of the calorimeter ranges from $6.0 \%$ to $7.5 \%$ for $1 \mathrm{MeV}$ electrons, while the time resolution is $250 \mathrm{ps}$.

The apparatus accommodates almost $10 \mathrm{~kg}$ of different, highly enriched $(95 \%-99 \%)$ double beta decay isotopes: ${ }^{100} \mathrm{Mo}(6914 \mathrm{~g}),{ }^{82} \mathrm{Se}(932 \mathrm{~g}),{ }^{116} \mathrm{Cd}(405 \mathrm{~g}),{ }^{130} \mathrm{Te}(454 \mathrm{~g}),{ }^{150} \mathrm{Nd}(34 \mathrm{~g})$, ${ }^{96} \mathrm{Zr}(9 \mathrm{~g})$, and ${ }^{48} \mathrm{Ca}(7 \mathrm{~g})$. Three sectors are also used for external background measurement and are equipped respectively with pure $\mathrm{Cu}$ (one sector, $621 \mathrm{~g}$ ) and natural $\mathrm{Te}(1.7 \mathrm{sectors}, 491 \mathrm{~g}$ ). All the sources are produced in the form of thin metallic or composite foils with a density of (30 60) $\mathrm{mg} / \mathrm{cm}^{2}$.

The detector is surrounded by a solenoidal coil which generates a vertical magnetic field of 25 Gauss inside the wire chamber for the electron-positron recognition. Moreover, the whole set-up is covered by two types of shielding against external $\gamma$-rays and neutrons.

Detailed description of the NEMO 3 detector and more information about the experiment are given in Ref. [1].

\footnotetext{
${ }^{1} \mathrm{LSM}=$ Laboratoire Souterrain de Modane; http: //www-lsm. in2p3.fr. The remaining cosmic ray flux in the laboratory is only 4.2 muons per $\mathrm{m}^{2}$ and per day.
} 
Table 1: Limits (in eV) on the effective neutrino mass $\left\langle m_{v}\right\rangle$ obtained from different theoretical calculations of nuclear matrix elements with $T_{1 / 2}^{0 v \beta \beta}>4.6 \times 10^{23}$ y for ${ }^{100}$ Mo and $T_{1 / 2}^{0 v \beta \beta}>1.0 \times 10^{23}$ y for ${ }^{82} \mathrm{Se}$.

\begin{tabular}{llll}
\hline \hline \multicolumn{2}{l}{ Nuclear matrix elements } & ${ }^{100} \mathrm{Mo}$ & ${ }^{82} \mathrm{Se}$ \\
\hline Shell model & Caurier 1996 [7] & & $<4.9$ \\
QRPA & Rodin 2005 [6] & $<2.7-2.8$ & $<4.1-4.5$ \\
QRPA & Šimkovic 1999 [7] & $<1.0$ & $<3.3$ \\
QRPA & Suhonen 2003 [8, 9] & $<1.1$ & $<2.8-4.2$ \\
QRPA & Stoica 2001 [10] & $<0.7-1.1$ & $<1.7-3.7$ \\
\hline \hline
\end{tabular}

\section{Measurement of the $2 v \beta \beta$ decay}

A candidate for a $\beta \beta$ decay is a two-electron event which is defined with the following criteria: two tracks coming from the same vertex in the source foils, the curvature of the tracks corresponds to a negative charge, each track has to be associated with a fired scintillator, and the time-of-flight has to correspond to the case of two electrons emitted at the same time from the same vertex. In addition a threshold of $200 \mathrm{keV}$ is applied on energy of each electron. In order to suppress background from ${ }^{214} \mathrm{Bi}$ decay inside the tracking detector, which is followed by a ${ }^{214} \mathrm{Po} \alpha$-decay, it is required that there is no delayed Geiger cell hit (delayed with respect to the event trigger by up to $700 \mu$ s) close to the event vertex.

After background subtraction, there is a total of $219,000 \beta \beta$ events selected in signal from ${ }^{100} \mathrm{Mo}$ and $2,750 \beta \beta$ events in signal from ${ }^{82} \mathrm{Se}$, both corresponding to 389 effective days of data collection. The signal-to-background (S/B) ratio reaches 40 for ${ }^{100} \mathrm{Mo}$ and 4 for ${ }^{82} \mathrm{Se}$. The determined half-life values of the $2 v \beta \beta$ decay are $T_{1 / 2}^{2 v \beta \beta}=[7.11 \pm 0.002(\text { stat }) \pm 0.54(\text { syst })]^{18} \mathrm{y}$ for ${ }^{100} \mathrm{Mo}$ (with a Single State Dominance decay) and $T_{1 / 2}^{2 v \beta \beta}=[9.6 \pm 0.3(\text { stat }) \pm 1.0(\text { syst })]^{19} \mathrm{y}$ for ${ }^{82} \mathrm{Se}$. These values are in agreement with previous measurement [2] but they have higher precision.

\section{First results on the $0 v \beta \beta$ decay search}

A complete study of the background in the $0 v \beta \beta$ window has been performed. The level of each background component has been directly measured from data using different analysis channels. In the case of ${ }^{100} \mathrm{Mo}$, the expected background in the energy window [2.8-3.2] $\mathrm{MeV}$ is $8.1 \pm 1.3$ and 7 events have been observed. As for ${ }^{82} \mathrm{Se}$, the expected background in the energy window [2.7 - 3.2] MeV is $3.1 \pm 0.6$ and 5 events have been observed. The dominant background during this first running period was due to radon from the air inside the laboratory, which penetrates the detector shielding into the wire chamber of NEMO 3. So, the limits at $90 \%$ C.L. obtained from data corresponding to 389 effective days of data collection are $T_{1 / 2}^{0 v \beta \beta}>4.6 \times 10^{23}$ y for ${ }^{100} \mathrm{Mo}$ and $1.0 \times 10^{23} \mathrm{y}$ for ${ }^{82} \mathrm{Se}$. These limits are about ten times higher than the previous limits obtained for these two isotopes [3, 凤]. The upper limits for the effective Majorana neutrino mass $\left\langle m_{v}\right\rangle$ range from 0.7 to $2.8 \mathrm{eV}$ for ${ }^{100} \mathrm{Mo}$ and from 1.7 to $4.9 \mathrm{eV}$ for ${ }^{82} \mathrm{Se}$ depending on the nuclear matrix elements (NME) used (see Table 1). 
Under hypothesis of gluino or neutralino exchange and using NME from Ref. [11], the limits obtained for the trilinear R-parity-violating supersymmetric coupling are $\lambda_{111}^{\prime}<1.6 \times 10^{-4}$ for ${ }^{100} \mathrm{Mo}$ and $3.0 \times 10^{-4}$ for ${ }^{82} \mathrm{Se}$. Assuming the hypothesis of the right-handed weak current, the half-life limits are then $T_{1 / 2}^{0 v \beta \beta}>1.7 \times 10^{23}$ y at $90 \%$ C.L. for ${ }^{100} \mathrm{Mo}$ and $3.8 \times 10^{23}$ y at $90 \%$ C.L. for ${ }^{82} \mathrm{Se}$. Using the NME calculations from Refs. [8, 12], the following corresponding upper limits on the coupling constant are obtained: $\lambda<2.5 \times 10^{-6}$ for ${ }^{100} \mathrm{Mo}$ and $\lambda<3.8 \times 10^{-6}$ for ${ }^{82} \mathrm{Se}$.

\section{Conclusions}

The NEMO 3 detector has been running reliably since February 2003. The $2 v \beta \beta$ decays of ${ }^{100} \mathrm{Mo}$ and ${ }^{82} \mathrm{Se}$ have been measured with very high statistics and with better precision than in previous measurements. The obtained two-electron energy sum spectrum, the single electron energy spectrum, and the angular distribution (distribution of the emission angle between the two electrons) are all in good agreement with Monte-Carlo simulations.

No evidence for the $0 v \beta \beta$ decay of ${ }^{100} \mathrm{Mo}$ or ${ }^{82} \mathrm{Se}$ has been found in data corresponding to 389 days. The limits at $90 \%$ C.L. are $T_{1 / 2}^{0 v \beta \beta}>4.6 \times 10^{23}$ y for ${ }^{100} \mathrm{Mo}$ and $T_{1 / 2}^{0 v \beta \beta}>1.0 \times 10^{23} \mathrm{y}$ for ${ }^{82} \mathrm{Se}$. During this running period (Phase I), radon was the dominant background at a level 3 times higher than the $2 v \beta \beta$ background for ${ }^{100}$ Mo. This type of background has been significantly reduced by a factor of $\sim 10$ with a radon trapping facility, which has been in operation since December 2004 (Phase II). The expected sensitivity at $90 \%$ C.L. after five years of data collection with reduced radon background will be then $T_{1 / 2}^{0 v \beta \beta}>2 \times 10^{24}$ y for ${ }^{100} \mathrm{Mo}$ and $T_{1 / 2}^{0 v \beta \beta}>8 \times 10^{23}$ y for ${ }^{82} \mathrm{Se}$, which corresponds to $\left\langle m_{v}\right\rangle<0.3-1.3 \mathrm{eV}$ for ${ }^{100} \mathrm{Mo}$ and $\left\langle m_{v}\right\rangle<0.6-1.7 \mathrm{eV}$ for ${ }^{82} \mathrm{Se}$.

A portion of this work has been supported by the Grant Agency of the Czech Republic (grant No. 202/05/P293).

\section{References}

[1] R. Arnold et al. (NEMO Collaboration), Nucl. Instr. and Meth. in Phys. Res. A 536 (2005) 79.

[2] Particle Data Group, K. Hagiwara et al., Phys. Rev D66 (2002) 010001.

[3] H. Ejiri et al., Phys. Rev. C63 (2001) 065501.

[4] R. Arnold et al. (NEMO Collaboration), Nucl. Phys. A636 (1998) 209.

[5] E. Caurier et al., Phys. Rev. Lett. 77 (1996) 1954.

[6] V.A. Rodin et al., Phys. Rev. C68 (2003) 044302; nucl-th/0503063.

[7] F. Šimkovic et al., Phys. Rev. C60 (1999) 055502.

[8] M. Aunola et al., Nucl. Phys. A643 (1998) 207.

[9] J. Suhonen et al., Nucl. Phys. A723 (2003) 271.

[10] S. Stoica et al., Nucl. Phys. A694 (2001) 269.

[11] A. Faessler et al., Phys. Rev. D58 (1998) 115004.

[12] J. Suhonen, Nucl. Phys. A700 (2002) 649. 